\title{
Correction to: Histone deacetylase inhibitor ITF2357 (givinostat) reverts transformed phenotype and counteracts stemness in in vitro and in vivo models of human glioblastoma
}

\author{
Francesco Marampon ${ }^{1}$ - Flavio Leoni ${ }^{2}$. Andrea Mancini ${ }^{3}$. llaria Pietrantoni ${ }^{4}$. Silvia Codenotti ${ }^{5}$. Letizia Ferella ${ }^{3,6}$. \\ Francesca Megiorni ${ }^{7}$. Giuliana Porro ${ }^{2}$ - Elisabetta Galbiati ${ }^{2}$. Pietro Pozzi ${ }^{2} \cdot$ Paolo Mascagni $^{2} \cdot$ Alfredo Budillon $^{8}$. \\ Roberto Maggio ${ }^{4}$ - Vincenzo Tombolini ${ }^{1}$ - Alessandro Fanzani ${ }^{5}$. Giovanni Luca Gravina ${ }^{3,6}$. Claudio Festuccia ${ }^{3}(1)$
}

Published online: 26 July 2019

๑) Springer-Verlag GmbH Germany, part of Springer Nature 2019

\section{Correction to: \\ Journal of Cancer Research and Clinical Oncology (2019) 145:393-409 \\ https://doi.org/10.1007/s00432-018-2800-8}

In the original publication, the 6th author's first name and the last name was inverted and incorrectly published. The correct author name is Letizia Ferella.

Publisher's Note Springer Nature remains neutral with regard to jurisdictional claims in published maps and institutional affiliations.

The original article can be found online at https://doi.org/10.1007/ s00432-018-2800-8.

Claudio Festuccia

claudio.festuccia@univaq.it

1 Department of Radiotherapy, Policlinico Umberto I, "Sapienza" University of Rome, Rome, Italy

2 Research Center, Italfarmaco SpA, Cinisello Balsamo, Milan, Italy

3 Radiobiology Laboratory, Department of Biotechnological and Applied Clinical Sciences, University of L'Aquila, Via vetoiossnc, Coppito II, L'aquila, Italy

4 Laboratory of Pharmacology, Department of Biotechnological and Applied Clinical Sciences, University of L'Aquila, L'aquila, Italy

5 Department of Molecular and Translational Medicine, University of Brescia, Brescia, Italy

6 Division of Radiation Oncology, Department of Biotechnological and Applied Clinical Sciences, University of L'Aquila, L'aquila, Italy

7 Department of Experimental Medicine, "Sapienza" University of Rome, Rome, Italy

8 Experimental Pharmacology Unit, Istituto Nazionale Tumori-IRCCS-Fondazione G. Pascale, Naples, Italy 